SCIREA Journal of Health http://www.scirea.org/journal/PM

September 5, 2021

Volume 5, Issue 3, June 2021

\title{
Index case testing, a strategy of improving HIV positivity yield among sexual partners and biological children of Newly diagnosed HIV positive clients in Minna, Nigeria
}

\author{
Chindo Ibrahim Bisallah ${ }^{1, *}$,Onyilo Ochigbo Michael ${ }^{1}$, Prince Obinna Anyanwu ${ }^{2}$ and \\ Bilkisu Sulaiman ${ }^{3}$ \\ ${ }^{1}$ Heart to Heart Clinic, General Hospital, Minna \\ 2 icap in Nigeria (Columbia University) \\ ${ }^{3}$ School of health Technology, Minna \\ *Corresponding author email: chindoibro@gmail.com
}

\begin{abstract}
Introduction: The Human immunodeficiency virus (HIV) Index case testing strategy involves the provision of HIV testing services (HTS) to sexual partners or family members of known HIV positive individuals (index clients). This approach was introduced in our facility in September 2019 aimed at identifying sexual partners of newly diagnosed HIV positive clients as a key intervention in identifying new HIV infection.

Objective: To assess HIV positivity yield among sexual partners and biological children of Newly diagnosed HIV positive clients.

Methodology: This is an interventional, non-controlled study, designed to assess the outcome of Index case testing in improving the detection of HIV infections. It involves the identification of sexual partners and biological children of newly diagnosed HIV positive
\end{abstract}


clients (Index case). The index case is then requested to notify his or her sexual partner(s) to avail themselves for HIV testing. Any of the elicited partner that reports or contacted are offered HIV counseling and testing. Biological children of the index case are also offered HIV Testing Services (HTS). A questionnaire was used to collect data from index cases and sexual partners by health care provider on demographic and sexual characteristics of participants following their consent. Data collected was entered into SPSS version 23. Data was analyzed using both Excel and SPSS version 23.

Results: A total of 448 individuals out of the 552 elicited partners were tested, giving a percentage of those elicited that were tested (448/522) of $85.8 \%$. out of the 448 partners that were tested 265 know their HIV status and were already on ARV medication. A total of 178 partners who never knew their HIV status were tracked and tested, and 48 tested positive, yielding a positivity rate $(48 / 178)$ of $27 \%$ which was statistically significant.

Conclusion: The HIV positivity yield using Index case testing strategy among sexual partners and biological children of newly diagnosed HIV positive Clients was significant and therefore of added value as a key intervention in diagnosing new HIV infections, thereby contributing towards the attainment of the first $95 \%$ of UNAIDS target.

Keywords: Human immunodeficiency virus, HIV testing services (HTS), Index case, Sexual partners and HIV positivity yield.

\section{Introduction}

The Human immunodeficiency virus (HIV) Index case testing strategy involves the provision of HIV testing services (HTS) to sexual partners or family members of known HIV positive individuals (index clients) who are at increased risk of HIV infection [1]. In index case testing, a person with confirmed HIV infection (index case) is asked to contact family members (children, spouse, sexual partners, siblings, and parents), requesting them to be screened for HIV and to see if they will accept an HIV test. This approach has proven to be a key intervention in diagnosing new HIV infections, enrolling and sustaining them on treatment and care.

The national HIV prevalence in Nigeria has increased exponentially over the years, from $1.8 \%$ in 1991 to $5.8 \%$ in 2001 and gradually declined to $3.1 \%$ in 2014 [2]. In 2018, the 
Nigeria AIDS Indicator and Impact Survey (NAIIS) conducted by the federal ministry of health revealed a HIV prevalence of 1.4\% among adults age 15-64 years, 1.8 among females and $1.0 \%$ among males. The prevalence of HIV among children age $0-14$ years was $0.2 \%$ [3]. HIV prevalence varied by zone across Nigeria, with the highest prevalence in South South Zone $(3.1 \%)$ and the lowest prevalence in North West Zone (0.6\%). Niger state has a HIV prevalence of $0.7 \%[3]$.

UNAIDS and the National Agency for the Control of AIDS estimate that there are 1.9 million people living with HIV in Nigeria [4]. However, the report of NAIIS survey 2018 indicated that only $46.9 \%$ of the adult population surveyed know their HIV status (5). It therefore means that many HIV infected clients remain unaware of their HIV status. This reinforced the fact that HIV Testing Service (HTS) is most times offered to the general population, with less emphasis on key population groups at higher risk than the general population. Recently, there is increasing focus on Key Population groups such as Men who have Sex with Men, People Who Inject Drugs, and female commercial sex workers (FCSW) on HIV testing services and provision. Sexual partners of HIV positive clients are another important highrisk group that needs tracking as they constitute a critical mass that must be reached with HIV testing services in order to attain HIV epidemic control. This group has been neglected either because they have not been thought of as a high-risk group or some form of reluctance by the HIV positive clients to disclose their sexual partners due to fear or stigma.

Achieving the UNAIDS vision 95-95-95 target to end HIV/AIDS by 2030 requires that 95 percent of those living with HIV are identified and offered Anti-Retroviral Therapy (6). To achieve the first 95 of UNAIDS target, the identification of PLHIV through HIV testing among sexual partners of HIV positive index cases is key to the success of this strategy [1].

Several research studies have explored contact tracing, partner counseling and referral services (PCRS) in reaching key population which includes sexual partners of HIV positive patients $(7,8,9)$. Identifying new infections with the focus of achieving the first $95 \%$ UNAIDS target continue to be challenging with only $30.1 \%$ of the population in Nigeria knowing their HIV status or having received their HIV results as at 2018 (5). Strategic HIV testing targeting High risk population can ensure improvement in positivity yield especially among the partners of HIV positive clients, hence the introduction of HIV indexing case testing in our facility. 
Index case testing, if found to be effective will serve as a Strategic approach in identifying and offering HTS to sexual partners of index HIV positive clients. This approach targeting specifically partners of HIV positive clients is important as an added intervention to reduce new infection rates toward ending HIV/AIDS pandemic by 2030. The objective of this study is to assess HIV positivity yield among sexual partners and biological children of Newly diagnosed HIV positive clients through Index case testing strategy.

\section{Methodology}

\section{Study setting and population}

Nigeria is located in sub-Sahara Africa with a population of over 140 million people and occupying a landmass of 923,768 square kilometers [10]. Niger state is located in the North central geopolitical zone of Nigeria with a population of 3,950,249 people according to the 2006 national population commission census [10]. The projected population of Niger state for 2016 was 5,556,247[11]. The study population were sexual partners of newly diagnosed HIV clients that were offered HIV counseling and testing at various HIV testing by entry points of General hospital, Minna from September 2019 to August 2020. This entry points include HCT unit at ART clinic, Immunology laboratory, Pediatric clinic and outreach services for those that could not get to the health facility. Index cases that were unwilling to contact their sexual partners were excluded from the study. Sexual partners elicited and reported to the various entry point were offered HIV testing services. Those that were found to be positive were immediately enrolled into treatment and care.

\section{Study design}

This is an interventional, non-controlled study design to evaluate the effect of Index case testing in improving the detection of HIV infections among newly diagnosed HIV positive adults and children.

\section{Inclusion criteria}

Sexual partners and the biological children of all index cases of newly diagnosed HIV positive clients enrolled into treatment and care from September 2019 to August 2020. 


\section{Exclusion criteria}

Index cases that were unwilling to disclose or contact their sexual partners for HIV testing services were excluded.

\section{Intervention}

Index case testing strategy was introduced in our facility in September 2019. This is a process that involves the identification of sexual partners of newly diagnosed HIV positive clients (Index case). The index case is then requested to notify his sexual partners to avail themselves for HIV testing at any of the entry point in the facility or outreach. Any of the elicited partner that reports are offered HIV counseling and testing and if found positive are enrolled into HIV treatment and care. This method of identifying previously undiagnosed cases and enrolling them into treatment and care through the index cases will facilitate the attaining of the first 95\% target of UNAIDS thus reducing the burden and transmission of HIV infection within the population.

During this process confidentiality of both the index case and the sexual partners were respected and a written informed consent was signed by both Index clients and sexual partners. Index cases that agreed on disclosure and referral but could not bring their partner to the facility for HTS were contacted by the health worker or health care provider. Through this process the sexual partner was notified of their exposure and potential risk to HIV infection and offered HTS without giving any information about the index cases. Mobile phone was used to track these sexual partners and those that could not be reached were traced through their addresses by index case officers of the facility.

\section{Declaration}

We the authors declare no conflict of interest with respect to the research, authorship and publication of this paper. 


\section{Authors' Contributions}

All authors contributed to writing, reviewing and editing the manuscript. CIB and OOM designed the study; CIB, OOM, POA and BS designed project concept and implementation; CIB and OOM contributed to data collection; and CIB, OOM, POA and BS contributed to data analysis. All authors have read and approved the final manuscript.

\section{Ethical consideration}

Ethical clearance to conduct the study was obtained from General hospital Minna, Ethics Committee for Research Involving Human Subjects. Informed consent was sought from the index cases and sexual partners.

\section{Data collection and analysis}

A questionnaire was used to collect data from index cases and sexual partners by health care providers on demographic and sexual characteristics of participants following their consent from September 2019 - August 2020. We adapted and modified a structured questionnaire from the HTS client intake form which was delivered by the index case health care provider during the meeting with the index case clients. Specific questions on demographic information and the sexual risk behavior of the index cases and the sexual partners served as a guide. Data collected was entered into SPSS version 23. Data was analyzed using both Excel and SPSS version 23. Simple proportions, tables and graphs were used to estimate the sero-prevalence of HIV among sexual partners of index cases. Chi square was used to measure the association between categorical variables. The p-value of results were compared with level of significance of 0.05 .

\section{Results}

From September 2019 - August 2020 a total of 634 index cases newly enrolled adults and children attending ART clinic in General Hospital Minna were requested to contact family members including children, spouse, sexual partners, siblings, and parents to see if they will accept an HIV test. About 383 index cases accepted to bring their partners, an acceptance rate of $(384 / 634)$ of $60.5 \%$. However, 363 index cases eventually brought partners for testing. A 
total of 522 partners were elicited by these index cases. 448 individuals out of the 552 elicited partners were tested, giving a percentage of those elicited that were tested (448/522) of $85.8 \%$. out of the 448 partners that were tested 265 know their HIV status and were already on ARV medication. A total of 178 partners who never knew their HIV status were tracked and tested, and 48 tested positive, yielding a positivity rate $(48 / 178)$ of $27 \%$.

Table 1. socio-demographic characteristics (Age, sex and relationship to the index case)

\begin{tabular}{|c|c|c|c|c|}
\hline Variables & $\begin{array}{l}\text { Total } \\
\text { tested }\end{array}$ & HIV negative & $\begin{array}{l}\text { HIV } \\
\text { positive }\end{array}$ & P value \\
\hline \multicolumn{5}{|c|}{ Age (years) } \\
\hline $1-9$ & 55 & 49 & 6 & \\
\hline $10-14$ & 15 & 11 & 4 & Fisher exact test $=12.50, p$ \\
\hline $15-19$ & 5 & 3 & 2 & value $=0.019$ \\
\hline $20-24$ & 6 & 4 & 2 & \\
\hline $25-49$ & 84 & 55 & 29 & \\
\hline $50+$ & 13 & 8 & 5 & \\
\hline \multicolumn{5}{|l|}{ Gender } \\
\hline $\begin{array}{l}\text { Male } \\
\text { Female }\end{array}$ & $\begin{array}{l}93 \\
85\end{array}$ & $\begin{array}{l}79 \\
51\end{array}$ & $\begin{array}{l}14 \\
34\end{array}$ & $\begin{array}{l}\chi \quad 2=14.03, p \text { value }= \\
0.001\end{array}$ \\
\hline \multicolumn{5}{|c|}{ Relationship to index cases } \\
\hline Husband & 54 & 43 & 11 & \\
\hline Wife & 44 & 11 & 29 & Fisher exact test $=46.09, \mathrm{p}$ \\
\hline Son & 32 & 29 & 3 & value $=0.001$ \\
\hline Daughter & 40 & 36 & 4 & \\
\hline Boyfriend & 7 & 7 & 0 & \\
\hline Girlfriend & 1 & 0 & 1 & \\
\hline Total & 178 & 130 & 48 & \\
\hline
\end{tabular}

The total number of people that tested positive was 48 , giving a positivity yield of $27 \%$ (48/178). Table 1 above shows that the HIV positivity yield increased with age of $60.4 \%$ 
(29/48) between the age of 25 - 49 years. The positivity rate was less between the ages of $15-$ 19 and $20-24$ years of $4.1 \%(2 / 48)$ each. This difference was found to be statistically significant with a $\mathrm{p}$ value of 0.019 . Further analysis revealed that there was a significant difference in positivity yield between males and females $\left(\chi^{2}=14.03\right.$, $p$ value $\left.=0.001\right)$. In this group, the positivity rate among females was $70.8 \%(34 / 48)$ compared to males of $29.16 \%(14 / 48)$, despite the fact that more males $(52.2 \%)$ were tested than females $(48.8 \%)$. Results also showed that there was a statistically significant differences in positivity yield among relations of positive cases to the index cases. Among this group, the positivity rate was high in wives of index cases of 60.4\% (29/48) compared to husbands of index cases of $22.9 \%(11 / 48)$. The least positivity yield among this group was boyfriends with a zeroprevalence rate $(0 / 48)$. Among children, the combined positivity rate of either son or daughter was $14.58 \%(7 / 48)$.

Table 2. Total number of partners that were tracked and tested by health facility entry point

\begin{tabular}{|c|c|c|c|c|c|}
\hline S.no. & $\begin{array}{ll}\text { Facility entry } \\
\text { point }\end{array}$ & Total tested & HIV negative & HIV positive & P value \\
\hline 1 & ART clinic & 89 & 57 & 32 & \multirow{5}{*}{$\begin{array}{l}\mathrm{P} \text { value }= \\
0.018\end{array}$} \\
\hline 2 & Laboratory & 21 & 15 & 6 & \\
\hline 3 & Pediatric clinic & 54 & 44 & 10 & \\
\hline 4 & outreach & 14 & 14 & 0 & \\
\hline Total & & 178 & 130 & 48 & \\
\hline
\end{tabular}

Table 2 above shows where partners of index cases were tracked and tested within and outside the health facility. There was a statistically significant differences in the positivity yield in relation to the facility entry point with a $\mathrm{p}$ value of 0.018 . Majority of those that tested positive were seen at the ART clinic with a positive rate of $66.6 \%(32 / 48)$, even though the attendance was higher through this entry point compared to others. This was closely followed with the pediatric clinic that had a positivity yield of $20.8 \%$ (10/48). The outreach service recording zero positive yield. 
Total number of partners that were tracked and tested by health facility entry point

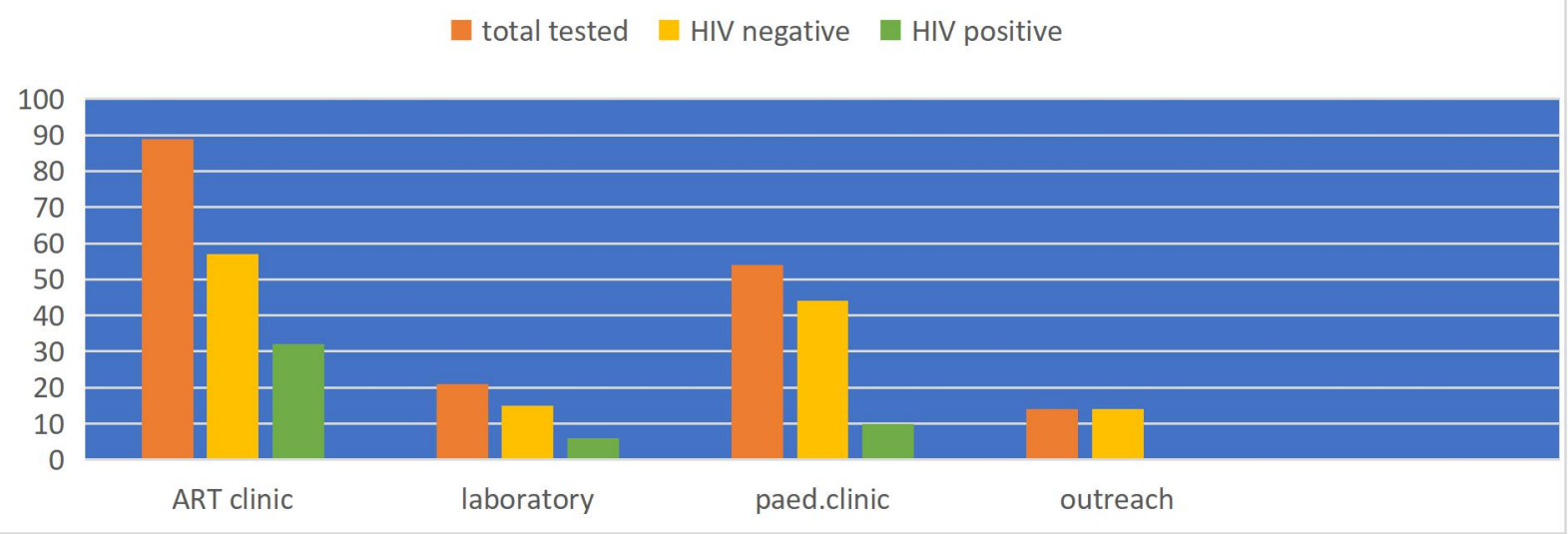

Figure 1. Distribution of HIV positive yield by health facility entry point.

Figure above shows the total number of partners that were tested, those negative and those that tested positive at different facility entry points. The ART clinic had the highest number of attendees, highest number of positive and negative cases with the outreach having the least all

cases.

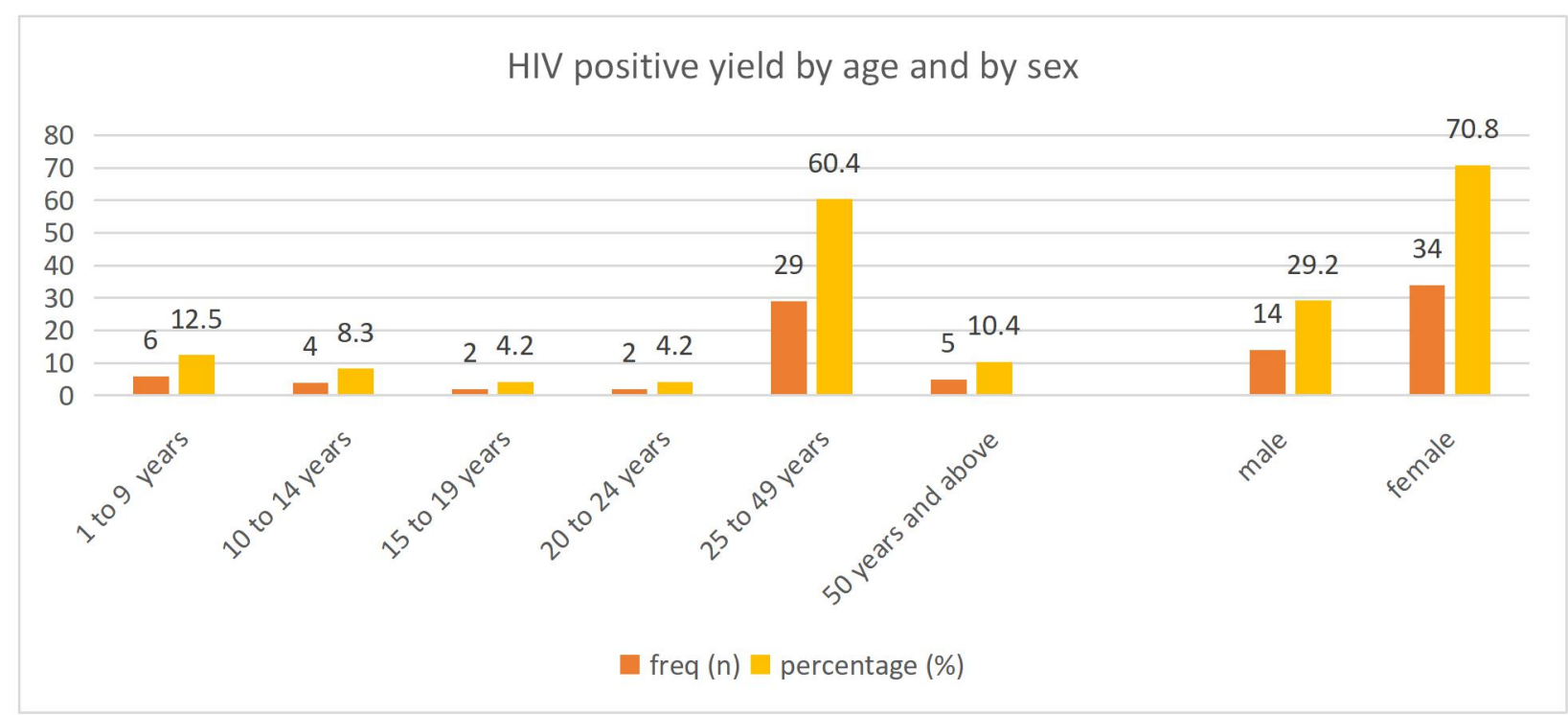

Figure 2: Distribution of HIV positive yield by age and sex

Figure 2 above shows a graphical display of the distribution of HIV positivity yield by age and sex. It shows that the highest HIV positivity yield was between the age range of 25- 49 years. It also shows that females had the highest positivity rate of $70.8 \%$ compared to males of $29.2 \%$. 


\section{Discussion}

Detecting new HIV infections continues to be challenging and grossly inadequate. According to Joint United Nations Programs on HIV/AIDS (UNAIDS) report in 2016, Nigeria had 220,000 new HIV infections and 160000 AIDS-related deaths. Additionally, an estimated 37,000 children were newly infected with HIV due to mother-to-child transmission. Out of the estimated 3.2 million people living with HIV in 2016, only $30 \%$ were accessing antiretroviral medications [12].

In Nigeria, $46.9 \%$ of persons living with HIV (PLHIV) aged 15-64 years were already aware of their HIV status (50.3\% among women living with HIV and 40.9\% among men living with HIV). This varied across age groups ranging from 31.0\% among young people aged 15-24 years to $52.8 \%$ among adults aged 35-49 years. Approximately 8 new cases of HIV infection occur annually per 10,000 adults. Overall, HIV prevalence among adults was $1.4 \%$, with $1.8 \%$ in women and $1.0 \%$ in men [3].

This study has shown a high yield of HIV positivity of up to $27 \%$ which is in agreement with the District Health System Strengthening and Quality Improvement for Service Delivery (DHSS) project which introduced index case testing in June 2016 in Malawi. In the case of the Malawi study, all patients attending ART clinics were asked to bring their family members for HIV testing organized at health facilities on days designated as family HIV testing days. Out of 25,572 adults and children from families living with HIV/AIDS tested through index case testing in 90 facilities, identified 5,528 people as HIV infected or HIV positivity rate of $22 \%$ [14]. In our study newly diagnosed HIV infected clients were our index cases in contrast to the Malawi project which instructed all patients to bring family members for testing. The Positivity yield was higher among those age 25 to 49 years, and females, consistent with the finding of the Malawi project. We observed that we had fewer women that were tested in our research yet the positivity yield was high than that of men.

Similarly, In Lesotho, 7,916 index cases in high burden ART facilities in 5 districts over a 2year period were approached for family members testing. A total of 10,854 individuals were tested (biological children, adolescents and sexual partners) and 454 were tested HIV positive. The overall HIV positivity rate was $4.2 \%$ [15]. This was in contrast to the findings of our study which yielded a positivity rate of $27 \%$. A Community Impact to Reach Key and Underserved Individuals for Treatment and Support (CIRKUITS) project in Zambia offered 
HIV Testing Services to 11,762 contacts of index clients and found 5260 HIV positive cases (positivity yield of $44.7 \%$ ) [16].

A study conducted in seven Local Government Areas of Federal Capital Territory (FCT), Abuja, Nigeria that targeted Index clients, who were PLHIV diagnosed from outreaches, hot spots and Provider Initiated Testing and Counseling (PITC) in comprehensive and primary health care facilities gave a positivity rate of 51\%. The study included both newly diagnosed PLHIV and those already on ART as identified from HIV testing source registers from October 2015 to July 2016. A total of 1277 index cases were counseled and interviewed with 879 index clients agreeing to disclosure. A total of 870 sexual contacts traced were tested for HIV, out of which 378 tested positives giving a positivity rate of 51\% (13). Comparing our finding with the above, one may appreciate why the positivity yield was much higher. A combination of both newly diagnosed HIV positive clients and those already on ART were used as index cases. Another study on the Positivity yield of HIV index testing services in Ondo State, Southwest Nigeria showed $26.9 \%$ HIV positivity rate in partners of male index clients [17]; which is in agreement with our study findings.

\section{Study Limitations}

The data collected was analyzed in aggregate. This limits the ability to conduct individuallevel evaluation of testing as well as socio-demographic factors associated with uptake of the HTS index testing. The study did not also evaluate the rate and reasons for index case refusals to contact sexual partners which may due to concerns about Gender based violence.

Despite these limitations, our study showed the importance of index testing as a sure means of reaching the UNAIDS 95-95-95 targets. Further research is needed on reasons for ICT refusals and to elucidate potential adverse effects of ICT as it relates to Gender Based Violence among index of persons living with HIV.

\section{Conclusions}

In conclusion, our study yielded a HIV positivity rate of $27 \%$. The positivity yield increased with age, with people between 25 to 49 years having the highest. Females had a higher positivity rate than males despite the fact that slightly higher number of males were elicited and tested. The HIV positivity yield was higher in ART clinic compared to other health 
facility entry points. Similarly, the positivity yield was higher among women identified as sexual partners of the index cases. This study has demonstrated that the use of index case testing for HIV case detection is a cost-effective way in reaching the first 95 target especially with the paucity of funds currently available for HIV programs.

\section{Reference}

[1] CDC (2018), Index case testing: a promising strategy for achieving HIV epidemic control, District health system strengthening and quality improvement for service delivery in Malawi, https://www.msh.org/sites/default/files/cdc_-_index_case_brief.pdf

[2] FMOH (2016), National guidelines for HIV prevention treatment and care, https://naca.gov.ng/wp-content/uploads/2017/08/National-HIVAIDS-Prevention-andTreatment- Guidelines_2016.pdf

[3] NAIIS (2019), Nigeria HIV/AIDS Indicator and Impact Survey, National summary sheet, Preliminary findings,

[4] UNAIDS 'AIDSinfo' (accessed August 2020)

[5] NAIIS (2018), Nigeria HIV/AIDS indicator and impact survey (NAIIS) 2018 technical report

[6] UNAIDS (2015), Understanding Fast-Track: accelerating action to end the AIDS epidemic by 2030

[7] Dalal S, Johnson C, Fonner V, Kennedy CE, Siegfried N, Figueroa C, et al. (2017), Improving HIV test uptake and case finding with assisted partner notification services. AIDS 31:1867-76.

[8] Lin H, He N, Ding Y, Qiu D, Zhu W, Liu X, et al. (2012). Tracing sexual contacts of HIV-infected individuals in a rural prefecture, Eastern China. BMC Public Health 12 (533).

[9] Broeckaert L, Haworth-Brockman M. CATIE. (2014) Available from: http://www.catie.ca/en/pif/fall-2014/you-may-have-come-contact-hiv-contact-tracingcanada.Cited 2021 January 10].

[10] Niger State Government. (2017). About Niger state: ministry of science and technology Minna, Niger state.

[11] National Bureau of Statistics [NBS] (2017). Demographic statistics bulletin: National Bureau of statistics 2017

[12] UNAIDS. unaids.org. 2016 Available from: http://www.unaids.org/sites/default/ files/media_asset/2016-prevention-gap-report_en.pdf. [accessed Jan 2021].

[13] Katbi M, Adegboye A, Adedoyin A, Yunusa F, Kayode G, Bello M, Dakum P (2018), Effect of clients Strategic Index Case Testing on community-based detection of HIV infections (STRICT study), International Journal of Infectious Diseases 74 (2018) 54-6

[14] Licy Khongonyowa, Erik Schouten, and Aziz Abdallah (2018), Index case testing: a promising strategy for achieving HIV epidemic control, Malawi: district health system strengthening and quality improvement for service delivery, technical brief 2018. 
[15] Jubilee M, Faith Park F J, Chipango K, Pule K, Machinda A, Taruberekera N (2019), HIV index testing to improve HIV positivity rate and linkage to care and treatment of sexual partners, adolescents and children of PLHIV in Lesotho, PLoS ONE 14(3): e0212762.

[16] Mwango LK et al. (2020) Index and targeted community-based testing to optimize HIV case finding and ART linkage among men in Zambia, Journal of the International AIDS Society, 23(S2):e25520, http://onlinelibrary.wiley.com/doi/10.1002/jia2.25520/full, https://doi.org/10.1002/jia2.255205123(S2):e25520

[17] A. Emeh, S.O. Usman et al (2021) Positivity yield of HIV index testing services from selected healthcare facilities in Ondo State, southwest Nigeria. African Journal of $\begin{array}{lllll}\text { Clinical and Experimental } & \text { Microbiology, } & \text { Vol. } 22 & \text { No. } \\ \text { https://www.ajol.info/index.php/ajcem/article/view/203073, } & 10.4314 / \text { ajcem. v22i1.13 }\end{array}$ 1. 